\title{
Phasing the GMT with a next generation e-APD dispersed fringe sensor: design and on-sky prototyping
}

\author{
Derek Kopon ${ }^{\mathrm{a}}$, Brian McLeod ${ }^{\mathrm{a}}$, Antonin Bouchez ${ }^{\mathrm{c}}$, Daniel Catropa ${ }^{\mathrm{a}}$, Marcos A. van Dam ${ }^{\mathrm{b}}$, Ken \\ McCracken $^{\mathrm{a}}$, Stuart McMuldroch ${ }^{\mathrm{a}}$, William Podgorski ${ }^{\mathrm{a}}$, Joseph D’Arco ${ }^{\mathrm{a}}$, Laird Close ${ }^{\mathrm{d}}$, Jared Males ${ }^{\mathrm{d}}$, \\ Katie Morzinski ${ }^{\mathrm{d}}$ \\ ${ }^{a}$ Harvard-Smithsonian Center for Astrophysics, 60 Garden Street, Cambridge, MA, 02138 \\ ${ }^{\mathrm{b}}$ Flat Wavefronts, 21 Lascelles St., Christchurch 8022, New Zealand \\ ${ }^{\mathrm{c}}$ GMTO Corporation, 465 N. Halstead Street, Suite 250, Pasadena, CA 91107 \\ ${ }^{\mathrm{d}}$ Steward Observatory, University of Arizona, Tucson, AZ 85721
}

\begin{abstract}
The GMT will be a 25.4-meter telescope consisting of 7 primary and secondary mirror segments that must be phased to within a fraction of an imaging wavelength in order to achieve diffraction limited performance. The need to use off-axis guide stars, coupled with the large $(350 \mathrm{~mm})$ segment gaps, makes the task of phasing the GMT uniquely challenging. To meet these requirements, the Acquisition, Guiding, and Wavefront Sensing system (AGWS) will use four J-band dispersed fringe sensors (DFS) to measure segment piston at each segment boundary. This DFS will use a novel array of doublet prisms, instead of grisms, to disperse segment boundary fringes with maximal throughput, minimal stray light, and no spurious diffractive orders. The DFS will also use a low noise SAPHIRA e-APD array reading out at $\sim 100 \mathrm{~Hz}$ in order to freeze atmospheric turbulence in the segment boundary fringe images. We will test a prototype of this DFS on the Magellan Clay 6.5 meter telescope using an adaptive optics corrected beam from the MagAO system. We present the design of the next generation phasing prototype.
\end{abstract}

Keywords: Active optics, adaptive optics, Giant Magellan Telescope, phasing, dispersed fringe sensor

\section{INTRODUCTION}

\subsection{The GMT AGWS}

The Giant Magellan Telescope (GMT) will be a 25.4-meter diameter telescope with a primary mirror consisting of seven 8.4 meter segments. The Acquisition Guiding and Wavefront Sensing system (AGWS) located near the GMT focal plane will use off-axis guide stars to make the measurements required to keep the optics of the GMT coaligned, phased, pointing in the correct direction, and conforming to the correct mirror shape ${ }^{1,2,3}$ (Figure 1). The AGWS will also perform target acquisition with a 30 arcsec imaging mode and Shack-Hartmann wavefront sensing for ground-layer adaptive optics (GLAO) operation. When the GMT operates in natural seeing or GLAO mode, the AGWS is the primary wavefront sensing system for the telescope. When operating in natural guide star AO mode (NGSAO), the AGWS is responsible for the initial phasing of the seven segment pairs. In laser tomography AO mode (LTAO), the AGWS keeps the telescope phased over the course of the observations. In LTAO mode, the AGWS also provides high frequency tiptilt correction.

The AGWS consists of four identical movable star probes located in a plane just before the telescope focal plane (Figure 2). Each star probe contains five selectable optical channels: four visible and one IR. The four visible channels are: a 30 arcsec FOV acquisition camera; a Shack-Hartmann wavefront sensor capable both of long time-scale active optics measurements and ground-layer wavefront sensing at $100 \mathrm{~Hz}$; a fast $(\sim 1 \mathrm{kHz})$ guide channel for natural guide star tip/tilt measurement during LTAO; and a 7-subaperture channel with pupil rotation for segment tip/tilt sensing. The IR channel is the J-band phasing sensor that is fed by a dichroic beamsplitter.

·derek.kopon@cfa.harvard.edu 


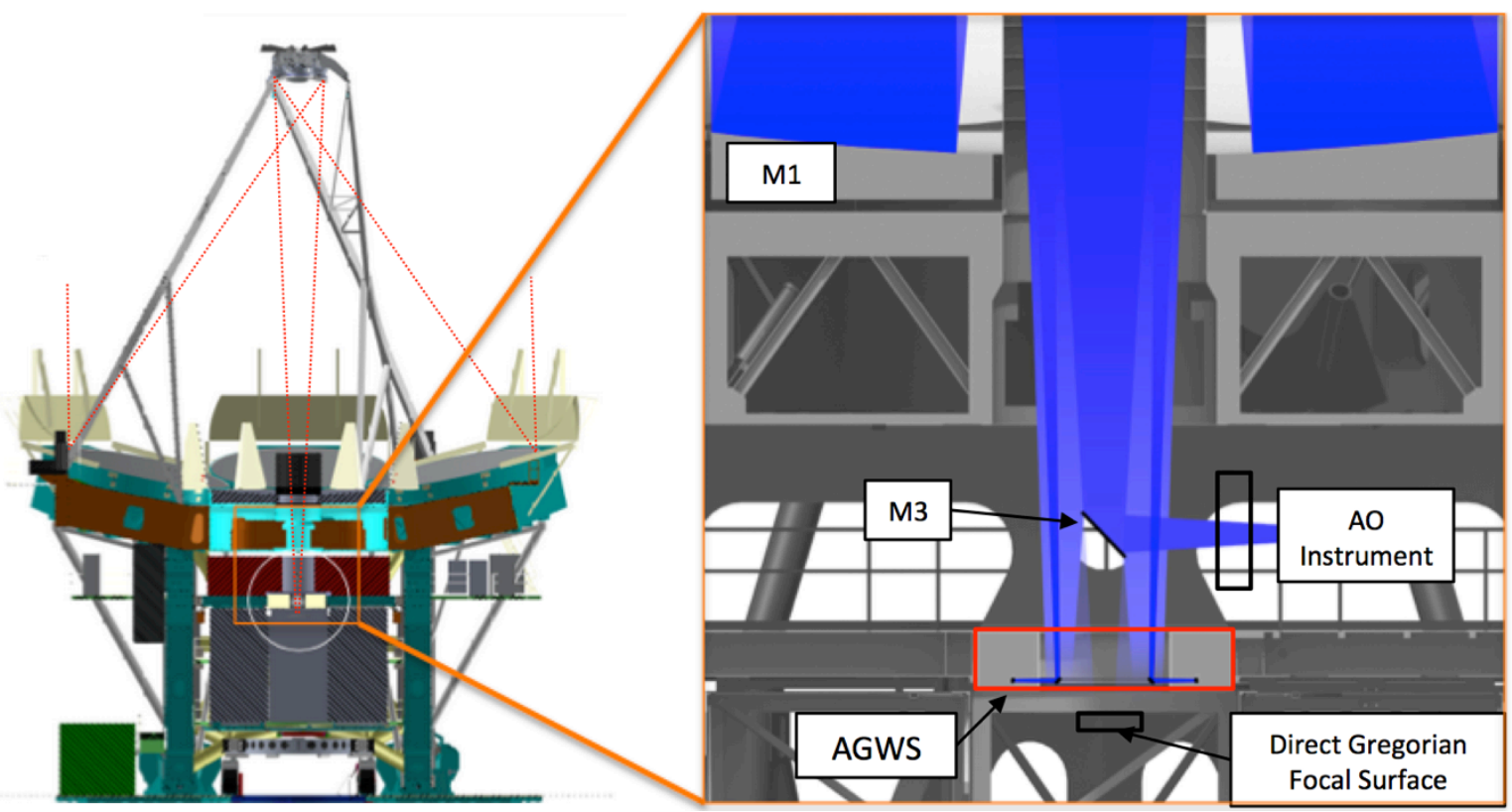

Figure 1: The AGWS location within the GMT.

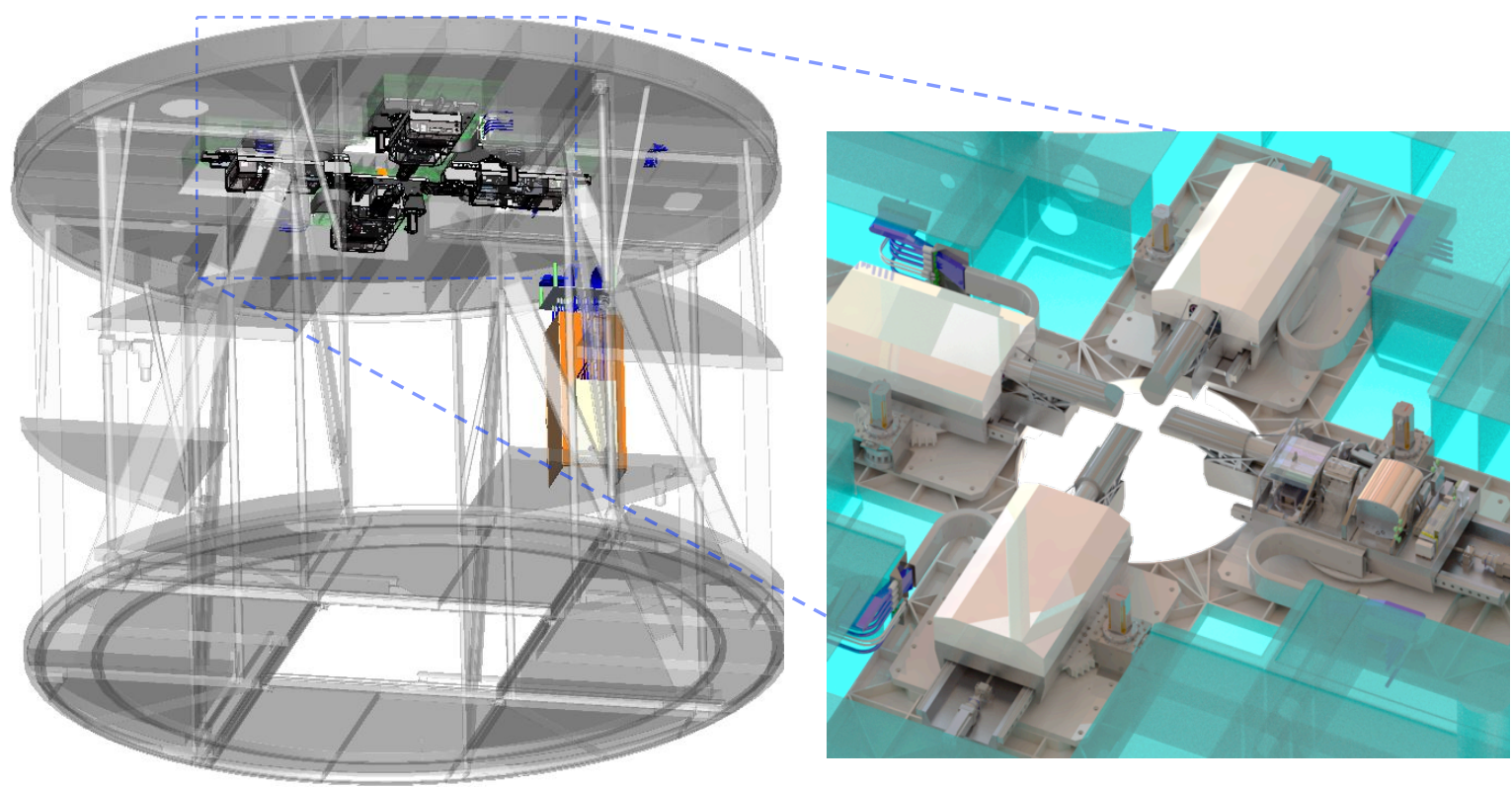

Figure 2: View of the AGWS mounted to the GMT Gregorian Instrument Rotator.

\subsection{The AGWS dispersed fringe phasing sensor}

Dichroic beamsplitters in each AGWS probe will allow visible light to be used for wavefront and fast tip/tilt sensing, while IR light is used for piston phase sensing. Simulations by van $\mathrm{Dam}^{4}$ show that the highest sky coverage for phasing the GMT will be achieved by using a J-band dispersed fringe sensor. The DFS operates by taking fringes produced at a segment boundary and dispersing them with a grism or prism in the direction perpendicular to the diffraction. Because the locations of the minima and maxima of the fringes of the diffraction pattern are wavelength dependent, the dispersed fringes will only be straight if the two segments are in phase. A phase shift between the segments will produce a tilt in 
the dispersed fringes. A detector reading out faster than the coherence time of the atmospheric seeing is then used to record images of the fringes. The Fourier transform of the fringes will give a peak whose vertical position is a measurement of the segment-to-segment phase difference (Figure 3).

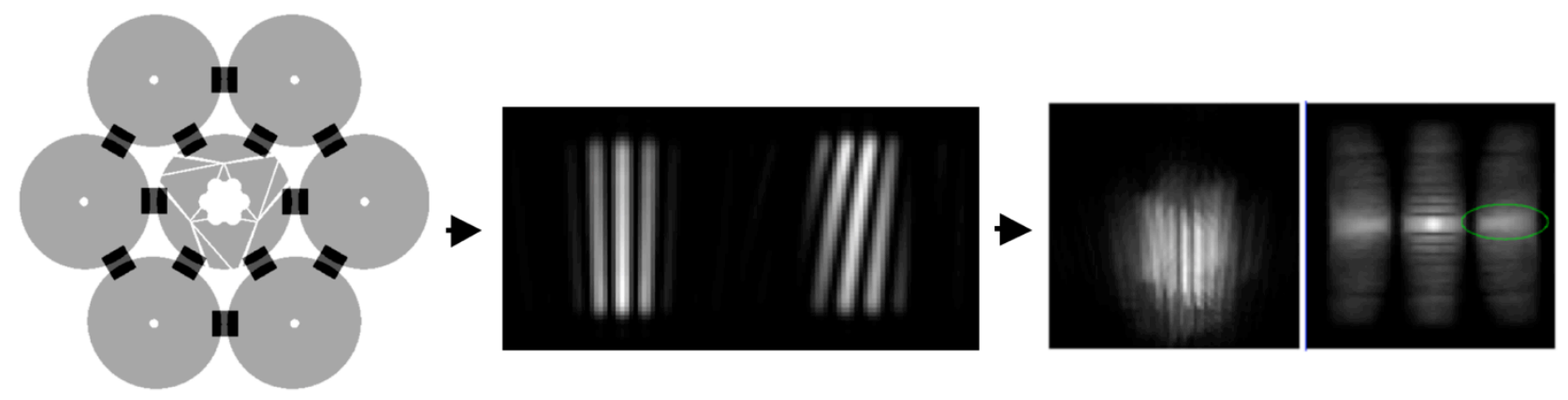

Figure 3: Left: The GMT pupil overlaid with $1.5 \mathrm{~m}$ square apertures at the segment boundaries. Center: Simulated fringes from one subaperture showing in phase (left) and a 10 micron phase shift (right). The dispersion direction is vertical.

Right: A simulated dispersed fringe image and its FFT. The vertical position of the circled peak is a function of the tilt of the fringes and thus a measure of the piston error.

\subsection{Previous phasing prototypes}

SAO has built and tested on sky two previous phasing prototypes. The first was a K-band dispersed Hartmann sensor that was tested at Magellan in $2012^{5}$. This instrument operated with a seeing limited beam from the F/11 secondary. The fringes were tip/tilt stabilized using a fast steering mirror. The advent of the SAPHIRA sub-electron read-noise fast readout IR array allows us to eliminate the need for tip/tilt stabilization.

The second phasing prototype was a dispersed fringe sensor operating behind the Magellan AO system. The sensor was tested on sky in December 2015,7. This sensor had two different wavelength channels: an I-band channel that used a Princeton EMCCD camera and a J-band channel that used a Ninox InGaAs detector. This prototype had a variable phase shifter and the ability to operate at 6 arcmin off-axis. This sensor verified that our DFS design can achieve our required 40 micron capture range of piston.

The lessons learned from the previous prototypes have informed the design of our most recent instrument, which will have a number of key modifications and improvements. The optical design uses custom optics that produce wellcorrected images. Lateral color in particular was carefully controlled, as this aberration produced phase biases in the previous prototype. A new phase shifter plate will give well qualified phase shifts in each of the three subaperture pairs. New off-axis lens optics will give us the ability to simulate the GMT off-axis aberrations. A prism doublet array will allow us to disperse fringes without the stray light and spurious diffractive orders generated by the grism disperser in the previous prototype. The SAPHIRA e-APD detector (commercially available in First Light Imaging's C-RED One camera) with a custom blocking filter should allow us fast sub-electron read-noise performance in the J-band.

\section{THE SAPHIRA DFS PROTOTYPE DESIGN}

\subsection{Prototype goals}

Our objective in designing the next generation phasing prototype is to build a sensor that is as similar as possible to the actual sensor design that we will use at the GMT and then to test it on sky under conditions that most closely mimic the conditions we expect at GMT. To that end, our optical design is identical to the sensor we have designed for GMT, which recently passed PDR. The only difference is the focal length of the collimating lens, which matches the F/16 Magellan beam instead of the F/8 beam of GMT. We have mechanically designed the mounts to reach the same alignment tolerances that we need to meet for the GMT sensor.

The patrol field in which the AGWS will acquire guide stars at GMT is 6-10 arcmin. We have therefore designed the prototype to operate both on-axis and at 6 arcmin off-axis. At GMT, the phasing sensors will be operated while AO is being performed with the on-axis science instrument. Therefore, the off-axis sensor will see the atmospheric structure produced by an on-axis AO-corrected beam. This beam will see disproportionately higher high order turbulence due to 
the isoplanatic error in the off-axis beam. The Magellan AO system will correct the beam using an on-axis guide star. The MagAO system uses a thin-shell adaptive secondary and a pyramid wavefront sensor. It is one of the closest AO analogues to the GMT design that is currently operational.

At an off-axis position, the Magellan and GMT telescopes produce different static aberrations. As described later, we will statically correct the Magellan aberrations and introduce the GMT aberrations. By adjusting the Magellan instrument rotator, we will also be able to change the direction of the parallactic angle relative to the aperture mask and prism dispersion in order to measure the effects of atmospheric dispersion on the phase measurements.

\subsection{Optical design}

The prototype optical design is an almost identical copy of the baseline GMT design (Figure 4), the only difference being the focal length of the first lens element (Figure 5). A collimating lens reimages the Magellan pupil onto a lasercut mask containing pairs of segment boundary apertures (Figure 6). The aperture pairs simulate a 1.5 meter square overlaid on the segment boundaries of the Magellan pupil for three different guide star off-axis distances: 6, 8, and 10 arcmin. Because the GMT primary and secondary segments are critically sized relative to each other, an increase in offaxis distance causes vignetting that effectively increases the inter-segment spacing.

After the mask, the prototype prism doublet array follows, which disperses the beam in the direction perpendicular to the diffraction pattern formed by the segment boundary apertures. After the prism array, a three doublet optical relay images the dispersed fringes onto the detector.

The prototype will have a pupil viewing channel to allow the aperture mask to be precisely aligned to the telescope pupil and to ensure that the apertures are uniformly illuminated. There will also be an "unobscured mode" that allows the prism and mask assembly to be removed from the beam and replaced with a lens assembly in order to allow the full telescope pupil to be viewed, or an undispersed/undiffracted image to be produced. The unobscured mode will be used to calibrate the detector using an on board flat field source.

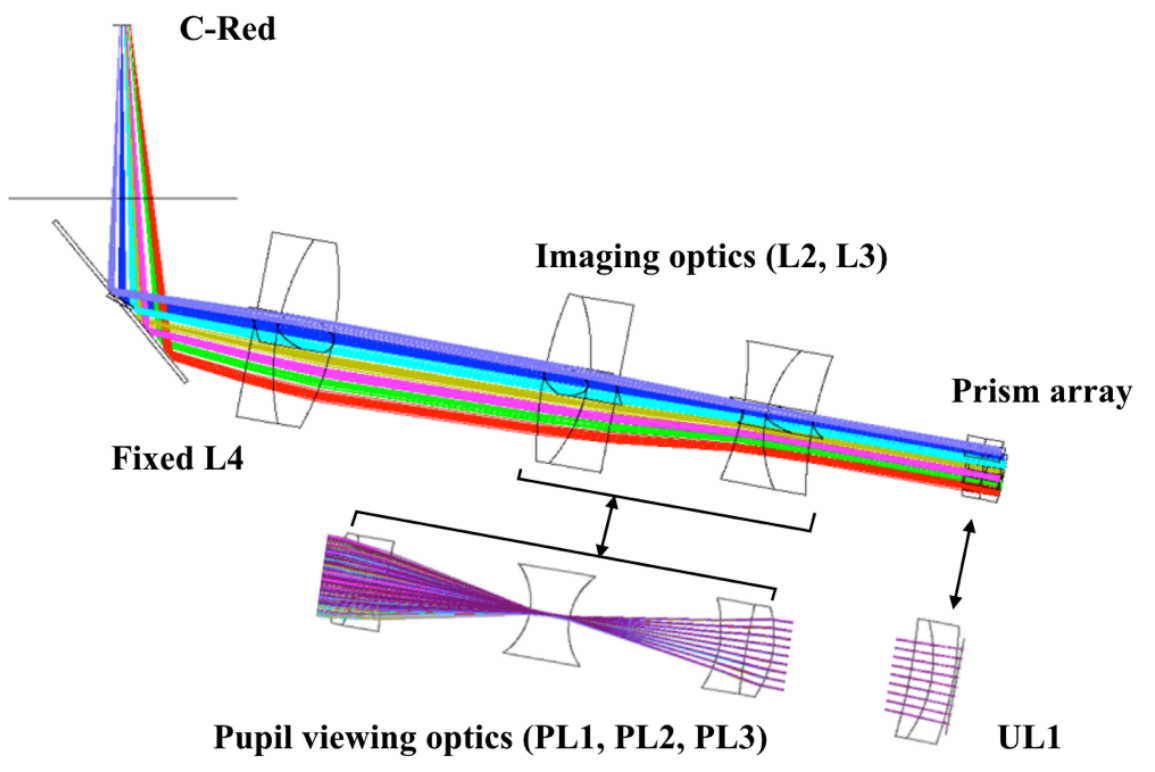

Figure 4: Layout of the phasing camera optics. A collimating lens (not shown) forms a pupil image on an aperture mask that is directly in front of the prism array. Dispersed light from the prism array is then reimaged onto the SAPHIRA detector by an optical relay. The UL1 and pupil viewing optics can be swapped into the beam as necessary to aid in alignment and calibration. 


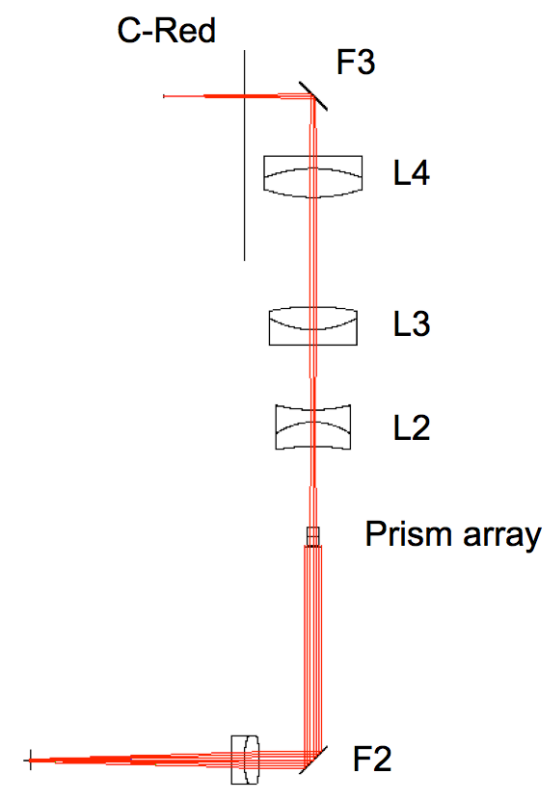

L1

Figure 5: Raytrace of the fringe viewing channel of the phasing prototype. The telescope focus is at the lower left. L1 collimates the beam and forms a pupil on the aperture mask just before the prism array. The downstream optical relay images the dispersed fringes onto the SAPHIRA detector.

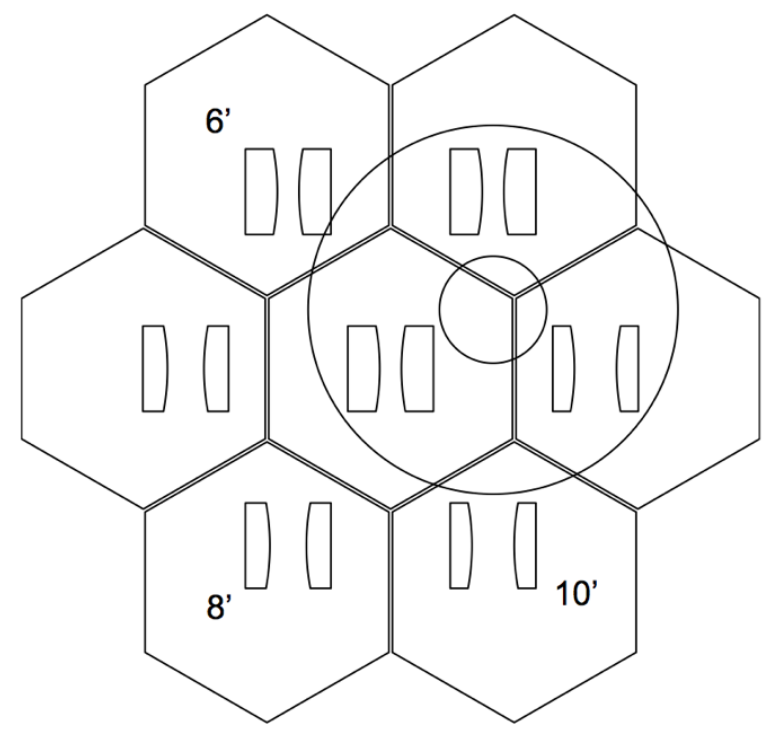

Figure 6: The pupil aperture mask overlaid with the circular Magellan pupil and the hexagonal prism array. Aperture pairs simulating GMT segment boundaries as seen by guide stars of different off-axis differences can be selected by moving the $\mathrm{prism} / \mathrm{mask}$ assembly relative to the beam. Three subaperture pairs can be positioned in the telescope beam at one time.

Figure 7 shows the layout of the phasing prototype, the light path through the instrument, and the location of the SAPHIRA detector. The Cal sources are located on a stage that can select either a white light point source, a flat field screen, or a 5 arcsec field stop. The light then continues to a reimaged pupil where the OAL, phase shifter, and prism select stages are located. Two different selectable optical relays reimage either the fringes or the pupil onto the SAPHIRA detector. 


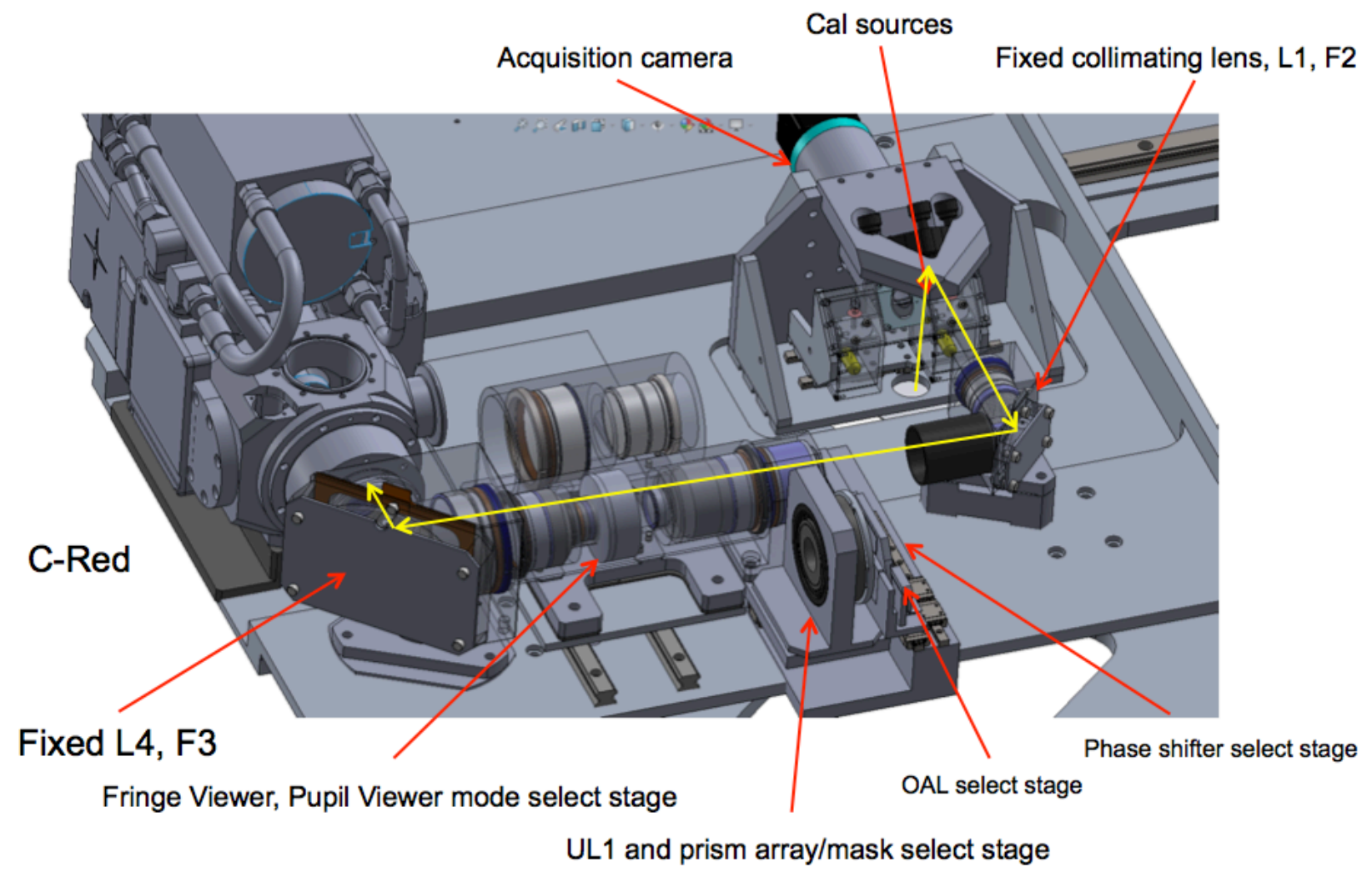

Figure 7: Layout of the phasing prototype. Light from the telescope comes out of the page through a hole in the plate near the Cal sources.

\subsection{Prism array prototype}

The complex pupil geometry of the GMT segment boundaries makes it difficult, if not impossible, to spatially filter the $0^{\text {th }}$ and $2^{\text {nd }}$ order diffraction patterns generated by any kind of grism array (Figure 8). To solve this problem, we have developed an array of prism doublets that use high dispersion optical glass to disperse the fringes generated by the segment subaperture pairs. Additionally, we have included a spherical surface on the second element of each doublet in order to eliminate the need for a separate lenslet with its associated throughput loss, optical registration difficulties, and expense. Precision Optical in Costa Mesa, CA is fabricating a 7-facet prototype array with the same material, size, and prism geometry as our GMT design (Figure 9). This array will be tested in the lab as a separate component to verify its fabrication tolerances and then installed in the phasing prototype for use at Magellan. 


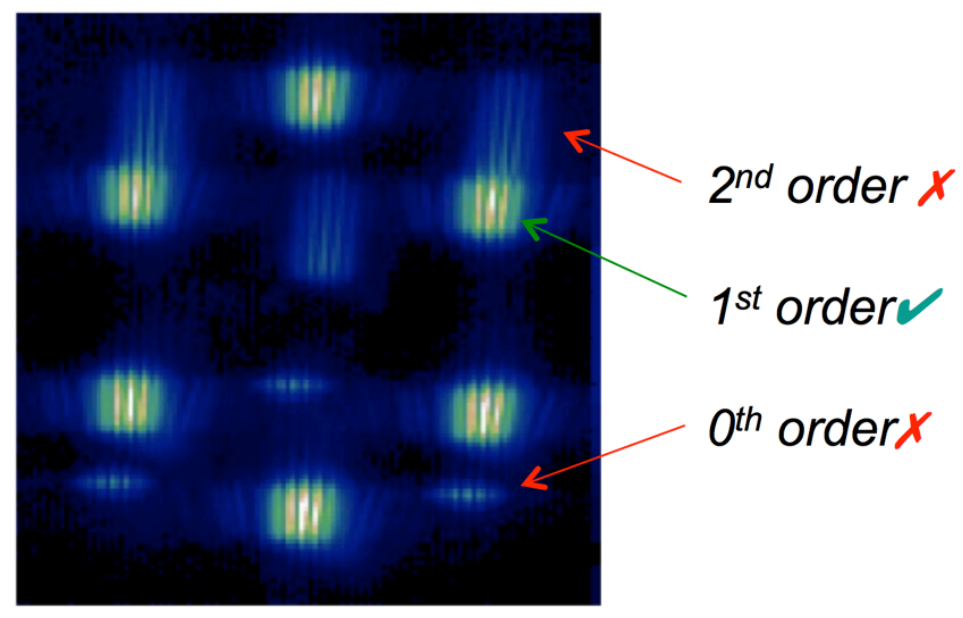

Figure 8: I-band laboratory image of the previous phasing prototype showing the extra diffractive orders produced by a grism. In a complex pupil geometry such as the GMT, these $0^{\text {th }}$ and $2^{\text {nd }}$ orders would overlap $1^{\text {st }}$ order images from other segment boundaries, creating higher noise and spurious signals. The prism array eliminates this issue.

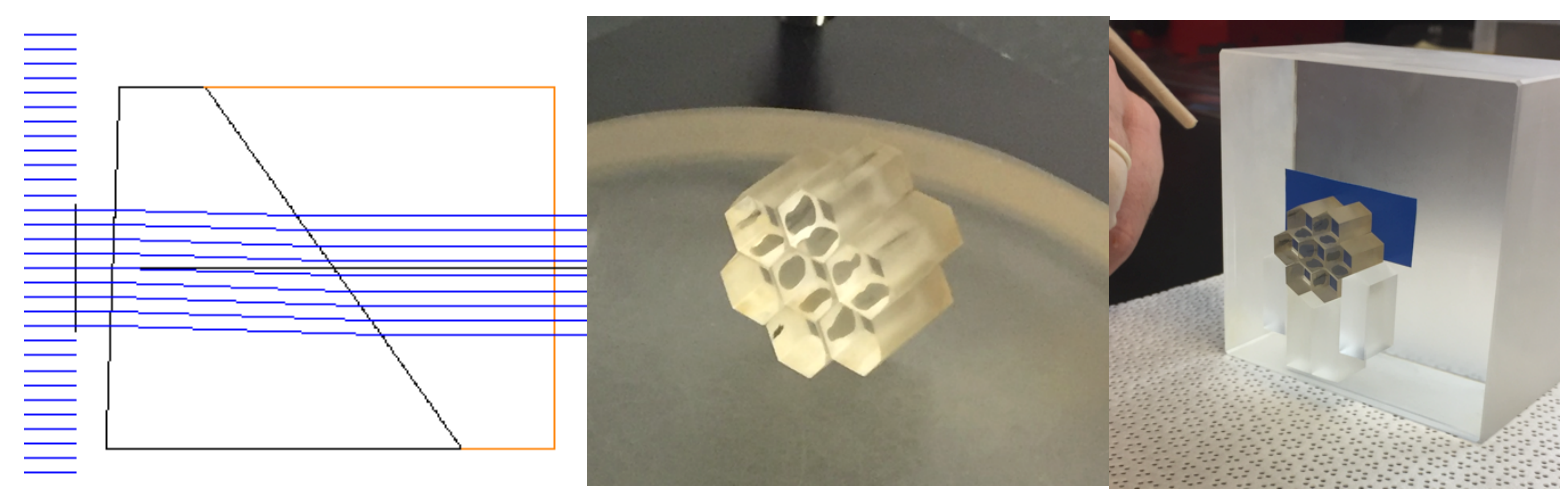

Figure 9: Left: Raytrace of one prism doublet facet. Light enters from the left and forms a pupil image on the aperture mask. The light then passes through the tilted front surface of the first element that is used to steer the beam. The last surface of the second element is a long-radius sphere. Right: Photographs of the array being fabricated in the shop.

\subsection{SAPHIRA array performance and blocking filter}

The key element in the phasing sensor that gives it high-sky coverage is the electron avalanche photodiode (eAPD) infrared SAPHIRA array produced by Leonardo (formerly Selex). This sensor operates in a charge multiplication mode that allows it to have an effective read noise less than 1e-. ${ }^{8}$ There are two characteristics that must be carefully managed to take advantage of the low noise.

First, as the gain is increased, the dark current also increases. We compute the optimum gain by considering the total readnoise+dark signal as a function of gain. Using data from G. Finger and First-Light Imaging, we estimate that the best performance will be obtained with an avalanche gain of approximately 50, which should yield an effective read noise of $0.3 \mathrm{e}-$, and dark noise of $0.7 \mathrm{e}$ - in a $10 \mathrm{~ms}$ exposure. Recent work by Finger, presented at this conference, has shown that the dark current can be reduced by operating the SAPHIRA with reduced bias voltages. With this reduction we may be able to operate at higher gains.

The second effect that must be managed is thermal background. The avalanche effect requires using a low-band gap layer of $\mathrm{HgCdTe}$ which is sensitive to light at thermal wavelengths. So though the detector layer has a cutoff wavelength $\lambda_{c}=2.5 \mu \mathrm{m}$, longer wavelength photons will pass through the detector layer and be absorbed in the gain layer 
which has has $\lambda_{c}=3.5 \mu \mathrm{m}$. At unity-gain the sensor has very high $\mathrm{QE}$ out to the cutoff wavelength of the gain layer. Because these photons may be absorbed part way through the gain layer, they will experience partial amplification, reducing the effective long-wavelength QE at high gain. ${ }^{8}$ Absent a blocking filter, our optical design would allow thermal photons in an $\mathrm{f} / 2$ cone to reach the detector. In addition, light coming in at larger angles will be scattered inside the camera and reach the detector. This light must be blocked by a cold filter inside the camera. Because the $300 \mathrm{~K}$ thermal background rises extremely steeply redward of the $3.5 \mu \mathrm{m}$ cutoff wavelength, the filter must block light out to $5 \mu \mathrm{m}$. We estimate the long wavelength QE of $\mathrm{HgCdTe}$ using the Urbach approximation. ${ }^{9}$ This extrapolation is shown in Figure 10. We have optimized the design of a baffle inside the C-RED camera using the FRED ${ }^{\circledR}$ scattered light software package. Multiplying the detector response curve by the black-body spectrum, the scattering function of the baffle, and a $10 \mathrm{x}$ reduction in long-wavelength $\mathrm{QE}$ for high-gain operation, yields the expected photon rates as a function of angle and wavelength (Figure 11). We require that the detector see thermal background summed over all wavelengths and incident angles of less than 10e-/pix/sec. This implies a very stringent blocking function on the filter (Figure 12). We have ordered a filter meeting this function from VIAVI.

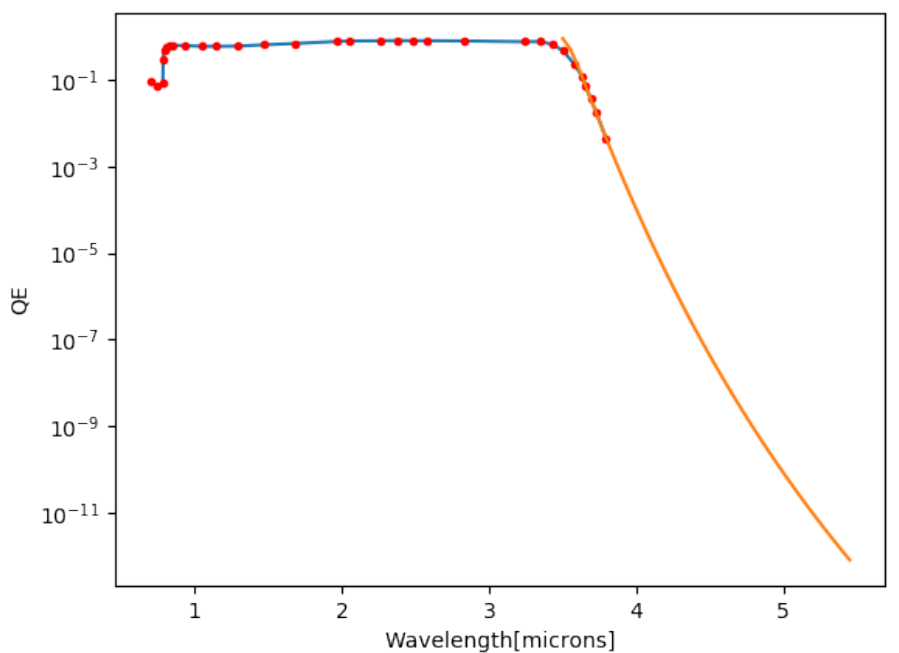

Figure 10: Extrapolation of SAPHIRA QE to longer wavelength using the Urbach approximation.

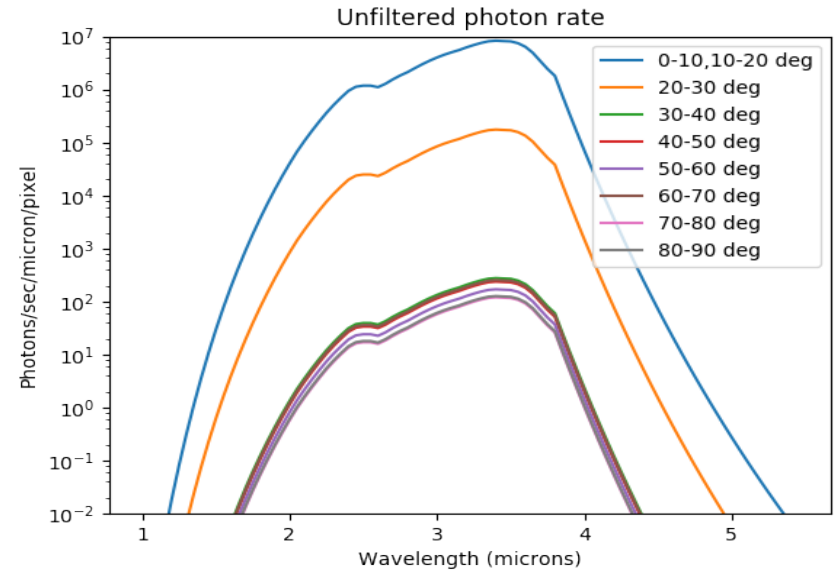

Figure 11: Photon rate as a function of incident angle that would be detected in the absence of a filter. We require that the sum of the integrals of all these curves be less than $10 \mathrm{e}-/ \mathrm{sec} / \mathrm{pix}$. 


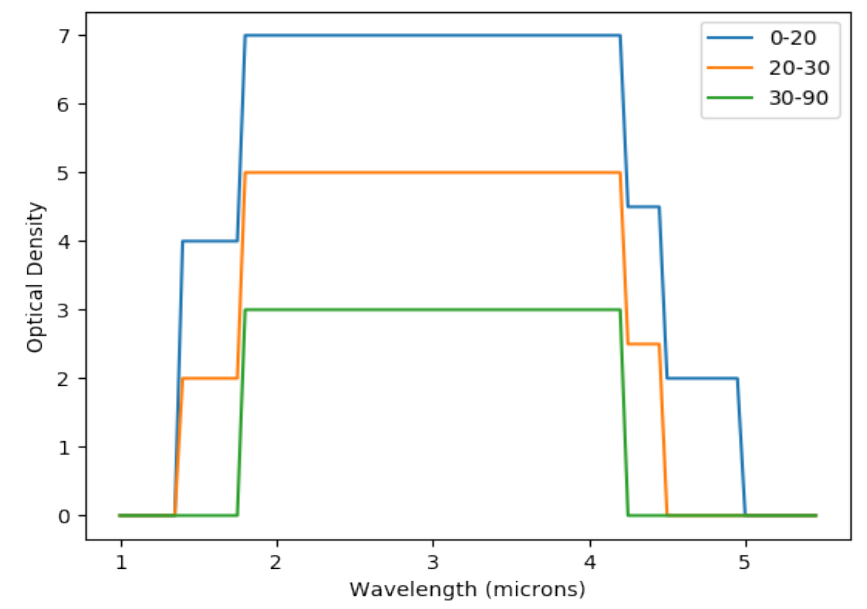

Figure 12: Filter blocking function that meets the $10 \mathrm{e}-/ \mathrm{sec} /$ pix requirement.

\subsection{Inter-segment phase shifts}

Because the Magellan 6.5 meter primary mirror is monolithic, the phasing prototype should measure zero phase shifts over each of the three subaperture pairs in the pupil. Once we had verified that the sensor does indeed measure zero phase, we will use a lithographically produced stepped phase plate to introduce discreet phase shifts into the beam. Our phase plate is a rectangular fused silica plate that can be translated in the beam to one of four discreet positions to give 200,400 , or $800 \mathrm{~nm}$ phase shifts in various combinations to each of the three subapertures in the beam.

\subsection{Off-axis aberration correction}

When operating off-axis, the Magellan telescope produces different aberrations than the GMT. To account for this difference, we will have two removable custom phase plates that can be introduced to the beam in front of the mask at the Prototype pupil. The first is the "Magellan OAL" (Off-Axis Lens), which will correct the Magellan telescope coma and astigmatism aberrations at 6 arcmin. The second is the "GMT OAL", which will introduce the astigmatism produced by the GMT at 8 arcmin off-axis. Both phase plates will be lithographically generated on $4 \mathrm{~mm}$ thick fused silica. Figure 13 shows the sag maps of the front surface of the two phase plates.
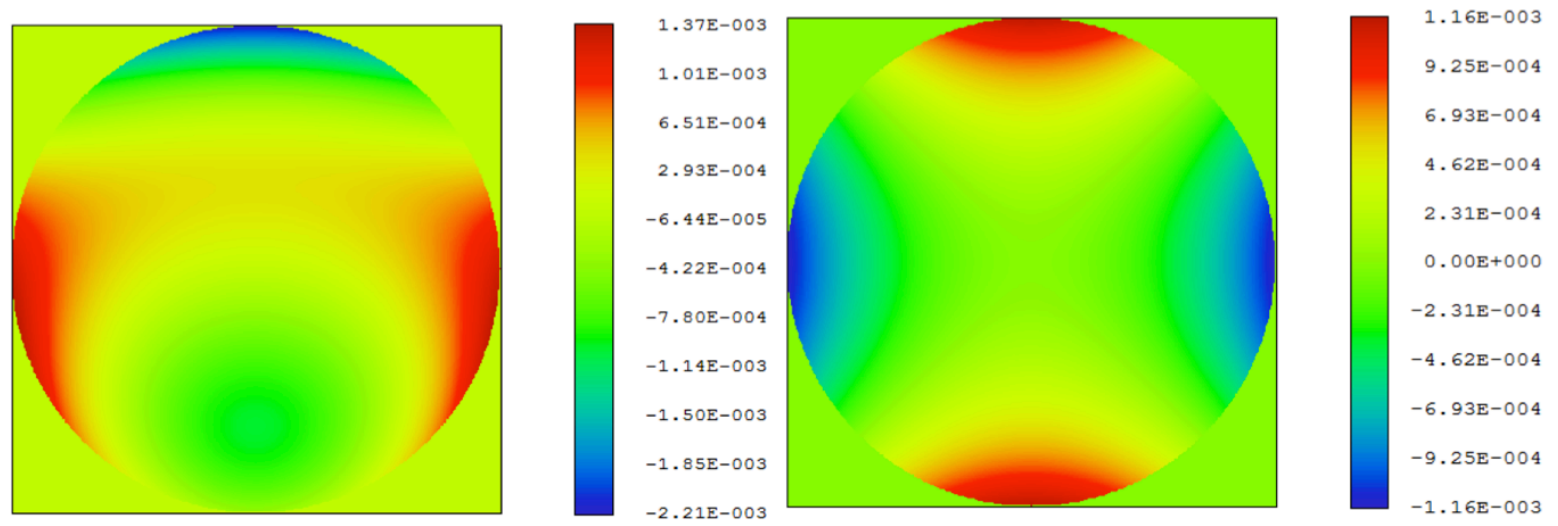

Figure 13: Left: "Magellan OAL" phase plate sag map that consists of coma and astigmatism aberration. Right: "GMT OAL" sag map that simulates the astigmatism produced by the GMT at 8 arcmin. 


\section{CONCLUSIONS}

We have completed the design and begun fabrication on a prototype dispersed fringe phasing sensor that will verify the critical technology needed to phase the GMT. This prototype uses several novel enabling technologies, such as a Saphira e-APD low noise IR detector with a custom cold filter, an array of high index prism doublets, and a custom optical design. By testing in an off-axis configuration behind an AO corrected beam from the MagAO instrument on Magellan, we will be able to replicate as closely as possible the conditions we will face at the GMT.

\section{Acknowledgements}

This work was supported by the GMTO Corporation, a non-profit organization operated on behalf of an international consortium of universities and institutions: Astronomy Australia Ltd., the Australian National University, the Carnegie Institution for Science, Harvard University, the Korea Astronomy and Space Science Institute, The Sao Paolo Research

Foundation, the Smithsonian Institution, The University of Texas at Austin, Texas A\&M University, University of Arizona, and University of Chicago.

\section{REFERENCES}

[1] McLeod, B., Bouchez, A. H., Espeland, B., Filgueira, J., Johns, M., Norton, T. J., Ordway, M., Podgorski, W. A., Roll, J., et al., "The Giant Magellan Telescope active optics system," Proc. SPIE 9145, 91451 T (2014).

[2] Quirós-Pacheco, F., Conan, R., McLeod, B., Irarrazaval, B.., Bouchez, A., "Wavefront control simulations for the Giant Magellan Telescope: Field-dependent segment piston control," AO4ELT4 Conf. Proc. (2015).

[3] Bouchez, A. H., McLeod, B. A., Acton, D. S., Kanneganti, S., Kibblewhite, E. J., Shectman, S. A.., van Dam, M. A., "The Giant Magellan Telescope phasing system," Proc. SPIE 8447, 84473S (2012).

[4] van Dam, M. A., McLeod, B. A.., Bouchez, A. H., "Dispersed fringe sensor for the Giant Magellan Telescope," Appl. Opt. 55(3), 539-547, OSA (2016).

[5] Kanneganti, S., McLeod, B. A., Ordway, M. P., Roll, J. B., Shectman, S. A., Bouchez, A. H., Codona, J., Eng, R., Gauron, T. M., et al., "A prototype phasing camera for the Giant Magellan Telescope," Proc. SPIE 8447, 844710-844752 (2012).

[6] Kopon, D., McLeod, B., van Dam, M. A., Bouchez, A., McCracken, K., Catropa, D., Podgorski, W., McMuldroch, S., Conder, A., et al., "On-sky demonstration of the GMT dispersed fringe phasing sensor prototype on the Magellan Telescope," Proc. SPIE 9909, 990911-990946 (2016).

[7] Kopon, D., McLeod, B., McCracken, K., van Dam, M., Bouchez, A., Conder, A., Podgorski, W. A., Catropa, D., McMuldroch, S., et al., "Prototyping the GMT phasing camera with the Magellan AO system," AO4ELT4 (2015).

[8] Finger, G., Baker, I., Alvarez, D., Dupuy, C., Ives, D. Mehrgan, L., Meyer, M., Stegmeier, J., \& Weller, H. J., "Sub-electron read noise and millisecond full-frame readout with the near infrared eAPD array SAPHIRA", Proc, SPIE 990912. (2016).

[9] Chang, Y., Guha, S., Grein, C. H., Velicu, S., Flatté, M. E., Nathan, V., \& Sivananthan, S., "Absorption of Narrow-Gap HgCdTe Near the Band Edge Including Nonprabolicity and the Urbach Tail", J. Electronic Materials, 36, 1000 (2007) 